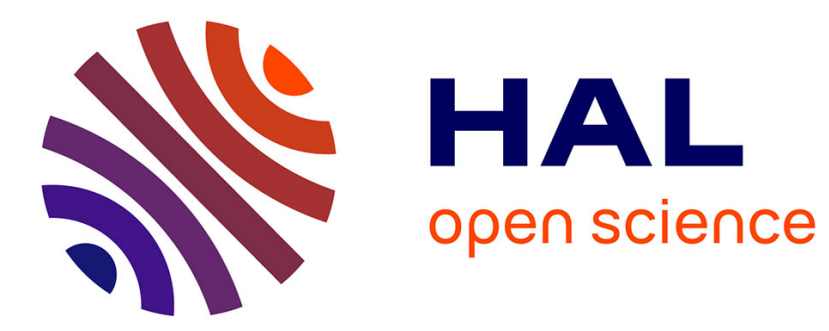

\title{
Direct Wavefront Measurement of Terahertz Pulses Using Two-Dimensional Electro-Optic Imaging
}

Mathilde Brossard, Harsono Cahyadi, Mathias Perrin, Jérôme Degert, Eric

Freysz, Takeshi Yasui, Emmanuel Abraham

\section{- To cite this version:}

Mathilde Brossard, Harsono Cahyadi, Mathias Perrin, Jérôme Degert, Eric Freysz, et al.. Direct Wavefront Measurement of Terahertz Pulses Using Two-Dimensional Electro-Optic Imaging. IEEE Transactions on Terahertz Science and Technology, 2017, 7 (6), pp.741-746. 10.1109/TTHZ.2017.2751249 . hal-01653645

\section{HAL Id: hal-01653645 \\ https://hal.science/hal-01653645}

Submitted on 1 Dec 2017

HAL is a multi-disciplinary open access archive for the deposit and dissemination of scientific research documents, whether they are published or not. The documents may come from teaching and research institutions in France or abroad, or from public or private research centers.
L'archive ouverte pluridisciplinaire HAL, est destinée au dépôt et à la diffusion de documents scientifiques de niveau recherche, publiés ou non, émanant des établissements d'enseignement et de recherche français ou étrangers, des laboratoires publics ou privés.

\section{(c)(1)(2)}

Distributed under a Creative Commons Attribution - ShareAlike| 4.0 International 


\title{
Direct Wavefront Measurement of Terahertz Pulses Using Two-Dimensional Electro-Optic Imaging
}

\author{
Mathilde Brossard, Harsono Cahyadi, Mathias Perrin, Jérôme Degert, \\ Eric Freysz, Takeshi Yasui, and Emmanuel Abraham
}

\begin{abstract}
We report on the development of a wavefront sensor for terahertz pulses using a direct two-dimensional electro-optic imaging system composed of a $\mathrm{ZnTe}$ crystal and a CMOS camera. By measuring the phase variation of the terahertz electric field in the crystal plane, we are able to reconstruct the terahertz ( $\mathrm{THz})$ wavefront in order to determine the optical aberrations of the optical system. Associated with deformable mirrors or specifically designed optics, the sensor will open the route to terahertz adaptive optics.
\end{abstract}

Index Terms-Optical aberrations, sensor, terahertz (THz), wavefront.

\section{INTRODUCTION}

$\mathbf{F}$ ROM the emergence of photonics technology, it has been forever essential to characterize and control the wavefront of optical beams, characterized by the surface of equiphase of the electromagnetic field, in order to determine the optical aberrations and optimize the performances of the optical systems. This can be accomplished for instance with the well-known Shack-Hartmann sensor which can locally measure the wavefront slopes of an incoming optical beam [1]. At present, in the visible and near-infrared spectral region, commercially available wavefront sensors can measure the optical aberrations and, associated with adaptive optics systems, they are able to correct these defaults in a wide variety of applications including astronomy, ophthalmology, and microscopy.

In the terahertz $(\mathrm{THz})$ spectral domain, it is still challenging to measure the spatial profile and wavefront of a $\mathrm{THz}$ beam due to the lack of effective $\mathrm{THz}$ two-dimensional (2-D) detectors. However, the wavefront characterization of $\mathrm{THz}$ beams is essential to optimize the spatial resolution or illumination uniformity of THz imaging systems, developed for a large variety of applications such as security and defense, nondestructive test-

Corresponding author: Emmanuel Abraham

M. Brossard is with the University of Bordeaux, CNRS, LOMA, UMR 5798, Talence F-33400, France, and also with NeTHIS-New Terahertz Imaging Systems, Mrignac 33700, France (e-mail: mathilde.brossard@ nethis-thz.com).

H. Cahyadi and T. Yasui are with the Graduate School of Science and Technology, Tokushima University, Tokushima 770-8506, Japan (e-mail: harsono@ femto.me.tokushima-u.ac.jp; yasui.takeshi@tokushima-u.ac.jp).

M. Perrin, J. Degert, E. Freysz, and E. Abraham are with the University of Bordeaux, CNRS, LOMA, UMR 5798, Talence F-33400, France (e-mail: mathias.perrin@u-bordeaux.fr; jerome.degert@u-bordeaux.fr; eric.freysz@ u-bordeaux.fr; emmanuel.abraham@u-bordeaux.fr). ing of industrial materials, food and pharmaceutical industry, communications, or cultural heritage [2]. Wavefront analysis of $\mathrm{THz}$ pulses is also mandatory for the control of far-field intensity distribution of time-domain spectrometers or to increase the peak power of intense terahertz sources and optimize the beam focalization of scanning $\mathrm{THz}$ time-domain imaging systems.

Previous studies were oriented toward the measurement of $\mathrm{THz}$ wavefronts for both continuous wave and pulsed sources. In 2008, Bitzer et al. determined the beam profiles of $\mathrm{THz}$ pulses after passing through a hyper-hemispherical silicon lens [3]. They observed an asymmetric spatiotemporal electric field dynamic at the focus of the lens attributed to a distortion of the incident $\mathrm{THz}$ wavefront. However, they did not measure the $\mathrm{THz}$ wavefront, but only the $\mathrm{THz}$ beam profile. Moreover, their indirect and time-consuming method used a $x y$-scanning system in order to determine point-by-point the beam profile. In 2012, Cui et al. proposed the first $\mathrm{THz}$ wavefront measurement associated with a Hartmann sensor and indirect $x y$-scanning method, detecting the radiation with a pyroelectric sensor [4], [5]. The same year, Richter et al. presented a similar arrangement with a 2-D detection consisting in a microbolometer camera [6]. However, their method cannot be applied with short $\mathrm{THz}$ pulses produced by femtosecond laser pulses owing to the low sensitivity of the thermal incoherent detector. Another aperture scanning device was proposed in 2013 to characterize the $\mathrm{THz}$ beam propagation in a time-domain spectrometer [7]. Associated with a Hartmann mask, it has been used to produce 2-D image of the $\mathrm{THz}$ wavefront. More recently, we proposed the wavefront characterization of $\mathrm{THz}$ pulses using a Hartmann sensor associated with a 2-D electro-optic (EO) imaging system [8]. We quantitatively determined the deformations of planar and converging spherical wavefronts using the modal Zernike reconstruction least-squares method. Nevertheless, our indirect method uses a mask which limits the beam transmission and requires an initial calibration or reference measurement.

In this paper, we propose a new direct method for the wavefront measurement of $\mathrm{THz}$ pulses. The principle of our measurement is as follows. It is well known that spatiotemporal profiles of $\mathrm{THz}$ transient electric fields can be directly determined through EO sampling in a nonlinear crystal [9]. By varying the time delay between a short optical laser probe pulse and the $\mathrm{THz}$ pulse, EO sampling can record the spatial dependence of the full (amplitude and phase) THz electric field, $E_{\mathrm{THz}}$, in the crystal plane. Then, from the phase variation of 


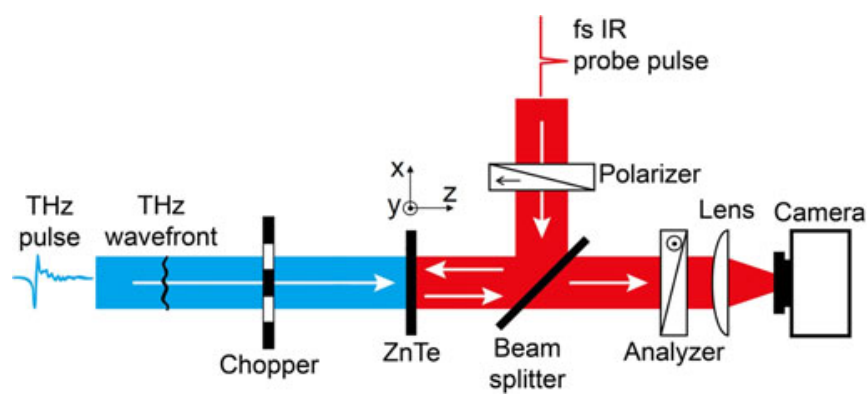

Fig. 1. Experimental setup of the direct THz wavefront sensor using 2-D EO imaging.

$E_{\mathrm{THz}}$ in this plane, we will demonstrate that a simple procedure makes it possible to determine the equiphase surface that is the wavefront.

\section{EXPERIMENTAL DETAILS}

For the demonstration of the method, broadband $\mathrm{THz}$ pulses were generated by optical rectification of amplified femtosecond laser pulses ( $800 \mathrm{~nm}, 1 \mathrm{~mJ}, 150 \mathrm{fs}, 1 \mathrm{kHz}$ repetition rate) in a $1 \mathrm{~mm}$-thick ZnTe crystal with a clear aperture of $25 \mathrm{~mm}$. The collimated THz beam is sent into another $1 \mathrm{~mm}$-thick ZnTe crystal for collinear EO detection with a time-delayed femtosecond laser probe pulse, reflected by the front left face of the crystal (see Fig. 1). There, owing to the Pockels effect, the spatial distribution of the broadband $\mathrm{THz}$ electric field $E_{\mathrm{THz}}(x, y)$ modifies the polarization distribution of the probe beam [9], whose intensity is finally captured by a $232 \times 232$ pixels CMOS camera (1000 fps) after passing through an analyzer and a lens that images the crystal plane. For a given time delay $t$ between the $\mathrm{THz}$ and the probe pulses, the system is able to provide a 2-D ( $x$-horizontal and $y$-vertical directions) image at $800 \mathrm{~nm}$ corresponding to the distribution of the $\mathrm{THz}$ electric field $E_{\mathrm{THz}}(x, y, t)$ at the crystal position. This image can be obtained with a $500 \mathrm{~Hz}$ acquisition rate, thanks to the synchronization with the optical chopper, the laser repetition rate, and a dynamic subtraction method [10]. However, signal-to-noise ratio higher than 100 usually requires an averaging time of $0.5 \mathrm{~s}$. By changing the time delay between the $\mathrm{THz}$ and probe pulses, it is possible to record the temporal evolution of the $\mathrm{THz}$ electric field $E_{\mathrm{THz}}(x, y, t)$. Thanks to this $\mathrm{THz}$ imaging system, we will demonstrate that we are able to fully analyze the spatial distribution of the THz electric field at the crystal position and finally determine the $\mathrm{THz}$ wavefront and extract the optical aberrations.

\section{RESULTS}

\section{A. Analysis of a Convergent $\mathrm{THz}$ beam}

First, to implement our method, we introduced a planoconvex THz lens ( $f=100 \mathrm{~mm}$ focal length) at $e=65 \mathrm{~mm}$ before the $\mathrm{ZnTe}$ crystal. This configuration provides a converging spherical $\mathrm{THz}$ beam at the crystal position with a theoretical $R=\left(f^{\prime}-e\right)+\left(\pi w_{0}^{2}\right)^{2} /\left(\Lambda^{2}\left(f^{\prime}-e\right)\right)=38 \mathrm{~mm}$ radius of curvature at $1 \mathrm{THz}$ assuming an incident Gaussian beam

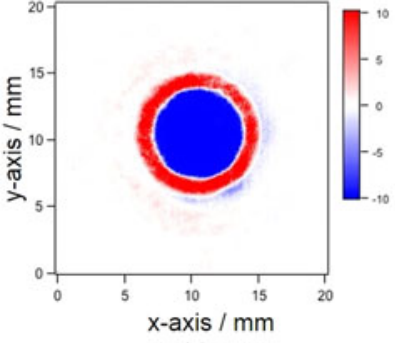

(a)

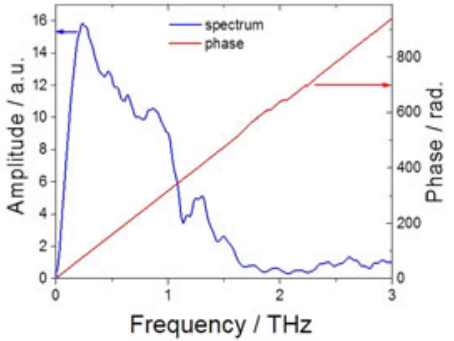

(b)
Fig. 2. Analysis of a convergent $\mathrm{THz}$ beam. (a) $E_{\mathrm{THz}}(x, y)$ for a $t=0 \mathrm{ps}$ time delay between the $\mathrm{THz}$ and laser probe pulses, from [8]. (b) Amplitude and phase of the complex electric field $E_{\mathrm{THz}}(\Omega)$, calculated for the central pixel of the image presented in (a)

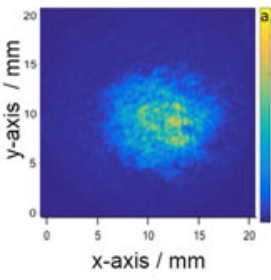

(a)

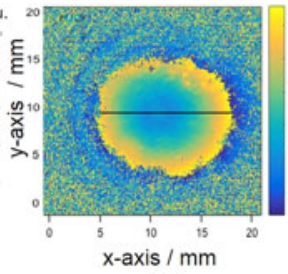

(b)

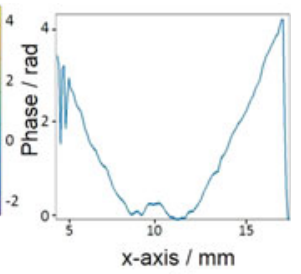

(c)
Fig. 3. Convergent $\mathrm{THz}$ beam. Amplitude (a) and relative phase (b) of $E_{\mathrm{THz}}(x, y, \Omega=1 \mathrm{THz})$ in the $\mathrm{ZnTe}$ crystal plane. (c) Evolution of the relative phase along the horizontal black line indicated in (b).

and a $w_{0}=1 \mathrm{~mm}$ beam-waist at the wavelength $\Lambda=300 \mu \mathrm{m}$. Fig. 2(a) displays the distribution of the converging spherical THz electric field, $E_{\mathrm{THz}}(x, y)$, for a $t=0$ ps time delay between the $\mathrm{THz}$ and laser probe pulses. This result is taken from [8]. However, in this paper, the $\mathrm{THz}$ wavefront reconstruction was made with the insertion of a Hartmann mask, whereas in the present work, we will show that a direct analysis of the 2-D EO imaging will provide the $\mathrm{THz}$ wavefront without any prior calibration. In Fig. 2(a), the central blue circle indicates a negative value of $E_{\mathrm{THz}}$, whereas the red ring is for a positive one, indicating the focusing of the $\mathrm{THz}$ beam at the crystal position. Then, by changing the time delay $t$ between the THz and probe pulses, we can record a set of 2-D EO transversal images representing the time-dependent amplitude $E_{\mathrm{THz}}(x, y, t)$, which is in fact a 3-D matrix whose analysis will provide us all the information we need for the wavefront reconstruction. The recording time of the whole 3-D matrix composed of 256 images is about $2 \mathrm{~min}$. It is clear that, for every pixels of the camera, i.e., for every $x$ and $y$ positions, it is possible to plot the corresponding temporal waveform $E_{\mathrm{THz}}(t)$. Then, through a Fourier transformation of the temporal data, it is possible to calculate the amplitude and the phase of the complex electric field $E_{\mathrm{THz}}(\Omega)$, where $\Omega$ is the $\mathrm{THz}$ frequency [see Fig. 2(b)]. The THz spectrum extends from 0 to $3 \mathrm{THz}$ with an expected linear dependence of the phase.

From the data presented in Fig. 2, it is straightforward to plot the amplitude and the phase of $E_{\mathrm{THz}}(x, y, \Omega)$ for every pixels of the camera, as shown for $\Omega=1 \mathrm{THz}$ in Fig. 3(a) and (b), respectively. For the sake of simplicity, the phase map at $1 \mathrm{THz}$ plotted in Fig. 3(b) does not represent the absolute phase 


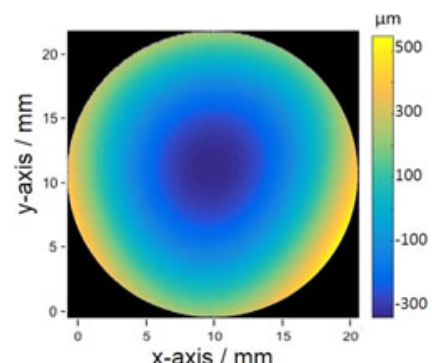

(a)

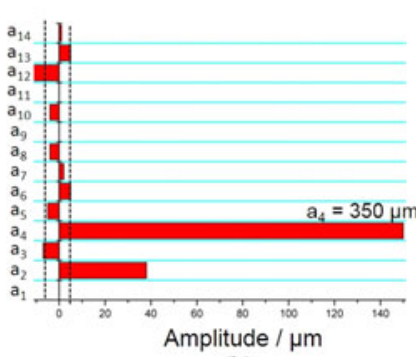

(b)
Fig. 4. Analysis of a convergent $\mathrm{THz}$ beam at $1 \mathrm{THz}$. (a) Reconstructed wavefront. (b) Amplitude of the Zernike coefficients. The dashed vertical black lines indicate the noise level or measurement accuracy. $a_{1}: y$-tilt, $a_{2}: x$-tilt, $a_{3}$ : astigmatism $45, a_{4}$ : defocus, $a_{5}$ : astigmatism 0/90, $a_{6}$ : vertical trefoil, $a_{7}$ : $y$-coma, $a_{8}: x$-coma, $a_{9}$ : oblique trefoil, $a_{10}:$ oblique quadrafoil, $a_{11}:$ oblique 2nd astigmatism, $a_{12}$ : spherical aberration, $a_{13}$ : vertical 2 nd astigmatism, $a_{14}$ : vertical quadrafoil.

of the beam, but the relative one with a zero value arbitrary fixed at the center. Moreover, in the following of the paper, we will restrict our analysis to $1 \mathrm{THz}$ but this latter can be easily generalized to every other frequency within $0-3 \mathrm{THz}$ spectral range, emphasizing the ability of our sensor to characterize broadband $\mathrm{THz}$ pulses. Next, the final reconstruction of the $\mathrm{THz}$ wavefront at the crystal position will simply use the values of the relative phase of $E_{\mathrm{THz}}(x, y, \Omega)$, represented by the phase map image in Fig. 3(b), as follows. First, we select a pixel $\left(x_{0}, y_{0}\right)$, e.g., the center of the phase map image, and we note the phase value $\phi_{0}\left(x_{0}, y_{0}\right)$ at this position. Then, except if the wavefront is perfectly planar without any optical aberrations, all the other pixels in the phase map image must have a different phase value. However, for every pixel position $(x, y)$, it is simple to calculate the distance $\Delta z(x, y)$ along the propagation axis for which the phase shift $\Delta \phi=2 \pi \Delta z / \Lambda$ will provide a new phase value being exactly $\phi_{0}$. The distribution of the resulting $\Delta z(x, y)$ values will design a new specific 3-D map, which will exactly correspond to the equiphase surface, demonstrating that our method is able to reconstruct the 3-D THz wavefront surface for every pixels of the camera.

To evaluate the accuracy of the method, we have to determine the minimal phase shift we can measure. Fig. 3(c) shows the evolution of the relative phase along the horizontal black line indicated in Fig. 3(b). The amplitude of the point-to-point fluctuations of the phase provides the minimum phase shift $\Delta \phi$ we can measure. For $\Omega=1 \mathrm{THz}$, we found $\Delta \phi=100 \mathrm{mrad}$, which corresponds to a minimum wavefront deformation of $(\Delta \phi \Lambda) /(2 \pi)=5 \mu$ m, i.e., $\Lambda / 60$. This wavefront measurement accuracy is comparable to standard commercial wavefront sensors designed for visible and infrared radiation.

First, the method is tested for the convergent $\mathrm{THz}$ beam, previously described in Figs. 2 and 3. Fig. 4(a) shows the reconstructed wavefront in the crystal plane with a quasi-spherical deformation from the center to the edge of the THz beam. The colored scale indicates the local wavefront deformation in $\mu \mathrm{m}$. However, the precise observation of this surface indicates that the wavefront is not only governed by a single defocus but also by other optical aberrations since the surface does not exhibit a perfect circular symmetry. To quantify these aberrations, the standard method consists in developing the wavefront surface $W(x, y)$ onto a linear combination of the Zernike polynomials [11]

$$
W(x, y)=\sum_{i} a_{i} Z_{i}(x, y)
$$

where $a_{i}$ represents the amplitudes of the polynomial $Z_{i}$, each polynomial corresponding to one specific optical aberration. Fig. 4(b) represents the amplitude of the 14 first Zernike polynomials. The main observation is obviously the strong defocus $a_{4}=350 \mu \mathrm{m}(1.17 \Lambda$ at $1 \mathrm{THz})$. To compare this value to the theoretical $R=38 \mathrm{~mm}$ radius of curvature of the $\mathrm{THz}$ beam at the sample position, we can assume a perfect spherical wavefront (i.e., $a_{i}=0, \forall i \neq 4$ ) and writes

$$
W(r)=\frac{r^{2}}{2 R}=2 \sqrt{3} a_{4} \rho^{2}
$$

where $r=\left(x^{2}+y^{2}\right)^{1 / 2}$ and $\rho=r / \alpha$. The parameter $\alpha$ is a normalization factor since the Zernike polynomials are defined for a maximum circular aperture with $\rho=1$. In our measurement, we have $\alpha=8.5$ corresponding to a $17 \mathrm{~mm}$ beam diameter. From (2), we calculate $R=30 \mathrm{~mm}$ which is about the theoretical value. The $20 \%$ deviation can be attributed to the imperfect spherical wavefront. Fig. 4(b) can actually quantify these additional aberrations with a $x$-tilt $a_{2}=38 \mu \mathrm{m}$ and a spherical aberration $a_{12}=-15 \mu \mathrm{m}$. All the other aberrations are of the order of the measurement accuracy $(5 \mu \mathrm{m})$, indicated by the dashed vertical black lines in the figure, and cannot be strictly considered as relevant in this experiment.

\section{B. Analysis of an $x$-Tilted THz Beam}

After this first demonstration of validity of the method with a spherical wavefront, we removed the focusing lens before the detection crystal and intentionally horizontally tilted the $\mathrm{THz}$ beam with respect to the laser probe beam so that these two beams interact noncollinearly in the crystal. The angle between the two beams has been precisely measured to be $\theta=3.9 \mathrm{mrad}$. We introduced this precise tilt because it constitutes another optical aberration that we can finely control, providing another test to verify the validity of our method. According to the relation

$$
W(r)=r \tan \theta=2 a_{2} \rho
$$

the $x$-tilt parameter we introduced in the crystal plane is then $a_{2}=90 \mu \mathrm{m}$. Fig. 5 shows the reconstructed wavefront and the amplitudes of the corresponding Zernike coefficients. As expected, the major optical aberration is the horizontal $x$-tilt with a calculated value $a_{2}=86 \mu \mathrm{m}$ very close to the actual tilt we intentionally introduced. This additional test validates once again our wavefront reconstruction method using 2-D EO imaging.

\section{Analysis of a Planar THz Beam}

Finally, a third experiment has been performed in order to determine the optical aberrations of the collimated $\mathrm{THz}$ beam 


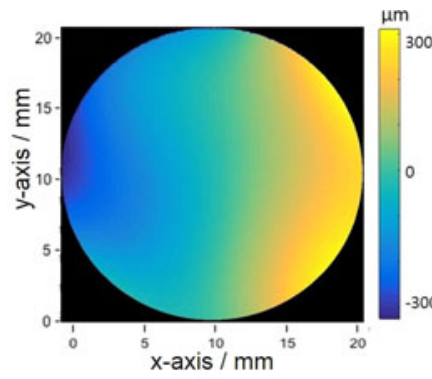

(a)

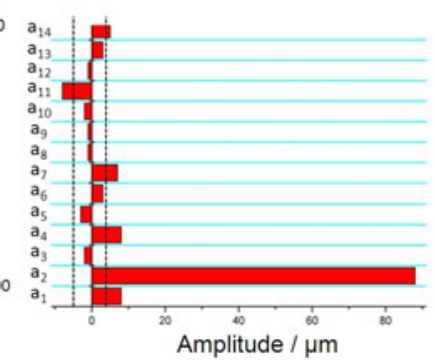

(b)
Fig. 5. Analysis of a $x$-tilted THz beam at $1 \mathrm{THz}$. (a) Reconstructed wavefront. (b) Amplitude of the Zernike coefficients. The dashed vertical black lines indicate the noise level or measurement accuracy.

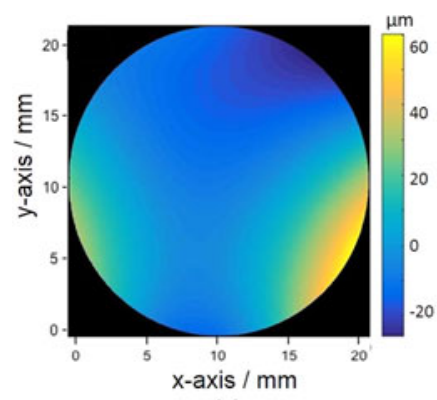

(a)

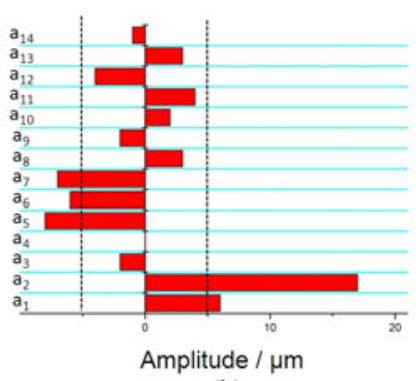

(b)
Fig. 6. Analysis of a planar $\mathrm{THz}$ beam at $1 \mathrm{THz}$. (a) Reconstructed wavefront. (b) Amplitude of the Zernike coefficients. The dashed vertical black lines indicate the noise level or measurement accuracy.

generated by optical rectification in the first $\mathrm{ZnTe}$ crystal. If the incident pump laser beam is supposed to have a planar wavefront, as illustrated in Fig. 1, a planar $\mathrm{THz}$ wavefront is also expected. Using our reconstruction procedure, Fig. 6 shows the wavefront and the amplitudes of the corresponding Zernike coefficients. Clearly, the THz wavefront is not exactly planar even if all optical aberrations are smaller than $20 \mu \mathrm{m}(\Lambda / 15)$. Besides the $x$ - and $y$-tilts, which are dependent on the angle between the probe and $\mathrm{THz}$ beams, the main optical aberration is the 0/90 astigmatism $\left(a_{5}=-9 \mu \mathrm{m}\right)$. This measurement is important since it indicates that the $\mathrm{THz}$ beam is not perfectly planar. Further investigations may concern the wavefront measurement of the incoming pump laser beam onto the $\mathrm{ZnTe}$ crystal using a commercially available wavefront sensor in order to determine if the $\mathrm{THz}$ wavefront deformation arises from the laser beam itself or from the $\mathrm{THz}$ generation process in the crystal.

\section{Comparison With the Hartmann Method}

As explained in the introduction, we also recently demonstrated the wavefront characterization of $\mathrm{THz}$ pulses using a Hartmann sensor associated with the similar a 2-D EO imaging system [8]. For this paper, the experimental setup was exactly the same as here, except that a Hartmann mask was inserted $10 \mathrm{~mm}$ before the detection crystal. Consequently, the $\mathrm{THz}$ wavefront was determined in the plane of the mask instead of the plane of the crystal, as it is the case for the present work. Therefore, if we want to compare the measurement presented in this pa-

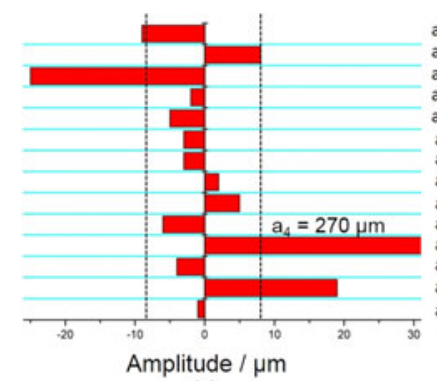

(a)

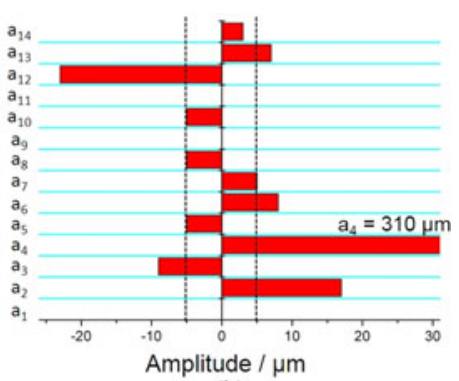

(b)
Fig. 7. Analysis of a convergent THz beam at $1 \mathrm{THz}$. (a) Zernike coefficients with the Hartmann method. (b) Zernike coefficients with the direct method.

per to that of [8], we need to propagate the THz electric field $10 \mathrm{~mm}$ before the crystal in order to reconstruct the THz wavefront at the same position. To this end, we have implemented a beam propagation method [12] to compute the field distribution at the position $z_{2}$, from the knowledge of the amplitude and phase of the field distribution at the position $z_{1}=z_{2}+10 \mathrm{~mm}$. The relationship between both scalar fields is computed using the Helmholtz equation in free space to obtain

$E\left(x, y, z_{2}\right)=\mathcal{F}^{-1}\left[E\left(k_{x}, k_{y}, z_{1}\right) \exp \left(\frac{k_{x}^{2}+k_{y}^{2}}{2 i k_{0}}\left(z_{1}-z_{2}\right)\right)\right]$

where $E\left(k_{x}, k_{y}, z_{1}\right)$ is the Fourier transform of $E\left(x, y, z_{1}\right)$, with $k_{i}$ the wave vectors and the symbol $\mathcal{F}^{-1}$ denotes the inverse Fourier transform. Note that the knowledge of both the amplitude and phase of the electric field at the position $z_{1}$ is necessary to back-propagate the field and compute $E\left(x, y, z_{2}\right)$. Based on the precedent equation, a freely available MATLAB program has been computed in order to propagate the THz waves [13]. This procedure makes it possible to compare both methods. However, we want to stress again that, the direct method we propose in the present paper only relies on the phase distribution of the $\mathrm{THz}$ electric field.

Let us recall that the Hartmann mask used in [8] consists of a 1-mm thick metallic plate with 9 by 9 circular holes $(1 \mathrm{~mm}$ diameter with $2 \mathrm{~mm}$ periodicity in the horizontal and vertical directions). The accuracy of the Hartmann method depends on the design of the mask (distance between two holes and size of the holes). In our case, we estimated that the accuracy was equal to $(\Lambda / 35)$ which is less precise than the direct measurement method proposed in this paper. In the case of the spherical convergent THz beam, we can see in Fig. 7 that the Zernike coefficients we obtain for the two methods are in good agreement. First, the amplitude of the defocus term is similar with $a_{4}=270 \mu \mathrm{m}$ with the Hartmann mask and $a_{4}=310 \mu \mathrm{m}$ with the direct method associated with the back propagation of $10 \mathrm{~mm}$. Note that the decrease of $a_{4}$, i.e., the increase of the radius of curvature, from the position $z_{1}$ to the position $z_{2}$, is consistent with the convergent spherical THz wave. Both methods also indicate similar amplitudes for $x$-tilt and spherical aberration. Only the direct method is able to measure the 45 astigmatism with $a_{3}=-9 \mu \mathrm{m}$, since the value is lower than the accuracy of the Hartmann method, indicated by the dashed vertical black lines in the figure. It is 
also important to notice that the Hartmann method measures the local slopes of the wavefront, which is finally calculated after integration of the data and application of a least-squares method [11]. Here, on the contrary, we directly measure the equiphase surface of the $\mathrm{THz}$ beam, which constitutes the wavefront.

\section{CONCLUSION}

In conclusion, by means of 2-D EO imaging of $\mathrm{THz}$ waveforms and subsequent calculation of the phase variation of the $\mathrm{THz}$ electric field, we were able to determine the wavefront with a precision of $\Lambda / 60$, without any prior reference measurement. We believe that our $\mathrm{THz}$ wavefront analyzer could provide an effective tool to control the generation of pulsed $\mathrm{THz}$ sources. Another interesting application concerns the association of the sensor with deformable mirrors in order to open the route to $\mathrm{THz}$ adaptive optics.

\section{ACKNOWLEDGMENT}

The authors would like to thank the referee of [8] whose useful comments and suggestions triggered this work.

\section{REFERENCES}

[1] R. V. Shack and B. C. Platt, "Production and use of a lenticular Hartmann screen," J. Opt. Soc. Amer., vol. 61, pp. 656-660, 1971.

[2] W. L. Chan, J. Deibel, and D. M. Mittleman, "Imaging with terahertz radiation," Rep. Prog. Phys., vol. 70, no. 8, pp. 1325-1379, Aug. 2007. [Online]. Available: http://stacks.iop.org/0034-4885/70/i=8/a=R02?key $=$ crossref.62dbc43c84616 2263df14f25f0c9bf17

[3] A. Bitzer, H. Helm, and M. Walther, "Beam-profiling and wavefrontsensing of THz pulses at the focus of a substrate-lens," IEEE J. Sel. Topics Quantum Electron., vol. 14, no. 2, pp. 476-481, 2008. [Online]. Available: http://ieeexplore.ieee.org/lpdocs/epic03/wrapper.htm?arnumber= 4481102

[4] M. Cui, J. N. Hovenier, Y. Ren, A. Polo, and J. R. Gao, "Terahertz wavefronts measured using the Hartmann sensor principle," Opt. Express, vol. 20, no. 13, pp. 14380-14391, 2012. [Online]. Available: https://www. osapublishing.org/abstract.cfm?uri=oe-20-13-14380

[5] M. Cui et al., " Beam and phase distributions of a terahertz quantum cascade wire laser," Appl. Phys. Lett., vol. 102, no. 11, 2013, Art. no. 111113. [Online]. Available: http://scitation.aip.org/content/aip/ journal/ap1/102/11/10.1063/1.4798250

[6] H. Richter et al., " Terahertz wavefront measurement with a Hartmann sensor," Appl. Phys. Lett., vol. 101, no. 3, 2012, Art. no. 031103. [Online]. Available: http://scitation.aip.org/content/aip/journal/apl/101/3/10. $1063 / 1.4737164$

[7] J. F. Molloy, M. Naftaly, and R. A. Dudley, "Characterization of terahertz beam profile and propagation," IEEE J. Sel. Topics Quantum Electron., vol. 19, no. 1, Jan. 2013, Art. no. 8401508. [Online]. Available: http://ieeexplore.iee.org/lpdocs/epic03/wrapper.htm?arnumber= 6224166

[8] E. Abraham et al., " Development of a wavefront sensor for terahertz pulses," Opt. Express, vol. 24, no. 5, pp. 5203-5211, Mar. 2016. [Online]. Available: https://www.osapublishing.org/abstract.cfm?URI=oe24-5-5203

[9] Q. Wu and X. Zhang, "Freespace electrooptic sampling of terahertz beams," Appl. Phys. Lett., vol. 67, no. 24, pp. 3523-3525, 1995.

[10] Z. Jiang, X. G. Xu, and X.-C. Zhang, "Improvement of terahertz imaging with a dynamic subtraction technique," Appl. Opt., vol. 39, no. 17, pp. 2982-2987, Jun. 2000. [Online]. Available: http://ao.osa. org/abstract.cfm?URI=ao-39-17-2982

[11] W. Southwell, "Wave-front estimation from wave-front slope measurements," J. Opt. Soc. Amer., vol. 70, no. 8, pp. 998-1006, Aug. 1980. [Online]. Available: http://www.osapublishing.org/abstract.cfm?URI=josa70-8-998

[12] A. E. Siegman, Lasers. Sausalito, CA, USA: Univ. Sci. Books, 1986.

[13] [Online]. Available: https://www.loma.cnrs.fr/mathias-perrin/
Mathilde Brossard was born in St. Etienne, France, in 1992. She received the Engineering degree from the Institut d'Optique Graduate School, Palaiseau, France, in 2015, and is currently working toward the Ph.D. degree on THz wavefront measurement at Bordeaux University, Bordeaux, France, in collaboration with the NeTHIS Company, Mérignac, France.

Harsono Cahyadi was born in Indonesia, in 1980. He received the Ph.D. degree from the Graduate School of Engineering Science, Osaka University, Japan, in 2013.

He continued as a Postdoctoral Researcher with the same laboratory until April 2014. He then joined the Institute of Technology and Science, The University of Tokushima, Tokushima, Japan. During his time with The University of Tokushima, he joined a research exchange program with Bordeaux University for the development of THz measurement systems. In May 2015 he became an Assistant Professor with the Department of Pathology and Cell Regulation, Kyoto Prefectural University of Medicine. He is currently a Senior Optics Engineer with a private company in Singapore where he is involved with the development of medical instrumentation based on optical spectroscopy. His research interests include various optical spectroscopy/microscopy techniques and biomedical optics.

Dr. Cahyadi is a member of the Optical Society of America, the International Society of Optics and Photonics, and the Spectroscopical Society of Japan.

Mathias Perrin is a CNRS Researcher involved in the field of nano-optics, plasmonics, guided (fiber) and nonlinear optics from a theoretical and numerical point of view. He participated in the development of quasi-normal mode theory to describe resonances in electromagnetism (from visible to $\mathrm{THz}$ ) and is currently involved with the modeling of phase masks for $\mathrm{THz}$ imaging.

Jérôme Degert received the Ph.D. degree in atomic and molecular physics from the University of Toulouse, Toulouse, France, in 2002.

In 2003, he was a Postdoctoral Research Fellow with the Max Planck Institute for Quantum Optics, Garching, Germany. During these years, he was involved in the field of optical pulse shaping and its application to quantum coherent control of ultrafast phenomena in simple atomic and molecular systems. From 2003 to 2016, he was an Assistant Professor with the University of Bordeaux, working with the Laboratoire Ondes et Matière $\mathrm{d}^{\prime}$ Aquitaine. He is currently a Professor His research interests range from the development of intense THz sources and their applications to nonlinear optics, THz pulse shaping and coherent control, to ultrafast time-resolved spectroscopy in the visible and $\mathrm{THz}$ ranges applied to the study of the optical properties of systems like spin crossover complexes and organic conducting polymers.

Eric Freysz received the Ph.D. degree in physics from Bordeaux University, Bordeaux, France, in 1983.

$\mathrm{He}$ is a Senior Researcher (DR1) with the CNRS and Deputy Director with the Department of Science and Technology, University of Bordeaux, Bordeaux, France. He joined the CNRS in 1984 after he earned the Ph.D. degree. He has authored or co-authored more than 130 publications in international journals, holds 14 patents, and has presented more than 220 conference proceedings. From 1991 to 1993, he was a Visiting Scientist with the Department of Physics, University of Berkeley, where he worked with Prof. Y. R. Shen. In 1994, he was a Invited Professor with the University of Pittsburgh, Pittsburgh, PA, USA. Since 1995, he has been the Director of different institutes and laboratory of physics in Bordeaux. He has a well-established expertise in lasers, nonlinear optics, linear and nonlinear $\mathrm{THz}$ spectroscopy, time resolved spectroscopy, and laser-induced phase transitions. 
Takeshi Yasui received the Ph.D. degree in mechanical engineering from Tokushima University, Tokushima, Japan, in 1997.

From 1997 to 1999, he was a Postdoctoral Research Fellow with the National Research Laboratory of Metrology, Japan. He was with the Graduate School of Engineering Science, Osaka University, Osaka, Japan, from 1999 to 2010, and was briefly with the University of Bordeaux I in 2007, and the University of Littoral Côte d' Opale in 2010, as an Invited Professor. He is currently a Professor with the Graduate School of Science and Technology, Tokushima University, and an Invited Professor with the Graduate School of Engineering Science, Osaka University. His research interests include $\mathrm{THz}$ instrumentation and metrology, nonlinear optical microscopy, and optical combs.

Prof. Yasui is a member of the Optical Society of America, the Japan Society of Applied Physics, the Optical Society of Japan, the Laser Society of Japan, the Japan Society of Medical Electronics and Biological Engineering, and the Japan Society of Mechanical Engineers. He was the recipient of the award for the Most Promising Young Scientist of the Optical Society of Japan in 1998, the Sakamoto Award of the Japan Society of Medical Electronics and Biological Engineering in 2006, the Optics Paper Award of the Japan Society of Applied Physics, and the Funai Award of the Japan Society of Mechanical Engineers in 2009.
Emmanuel Abraham was born in Reims, France, in 1970. He received the Ph.D. degree in physics from Bordeaux University, Bordeaux, France, in 1997. From 1997 to 1998, he was a Postdoctoral Research Fellow with the National Research Laboratory of Metrology, Tsukuba, Japan, and the FemtoSecond Technology project to study industrial applications of femtosecond lasers, including ultrafast optical Kerr gates. From 1998 to 2013, he was an Assistant Professor with Bordeaux University, working with the Laboratoire Ondes et Matière $\mathrm{d}^{\prime}$ Aquitaine (LOMA). He joined Osaka University as an Invited Researcher in 2007 and the University of Tokushima as a Short-Term Invited Fellow Researcher of Japan Society for the Promotion of Science in 2010. Since 2013, he has been a Professor with LOMA, Bordeaux University. He developed intense terahertz sources and studied their applications for nonlinear spectroscopy. Due to the international collaboration with Tokushima University, he also developed innovative instrumentation for $\mathrm{THz}$ science and technology. He has authored or co-authored more than 50 peer-reviewed papers, 4 patents, 4 book chapters, and more than 100 conference proceedings. His research interests include femtosecond time-resolved spectroscopy and nonlinear optics. He also has investigated the potential of terahertz radiation for the analysis of objects related to cultural heritage such as paintings, sculptures, ceramics, etc., by using pulsed and continuous millimeter-wave sources for 2-D imaging and 3-D terahertz computed tomography. 